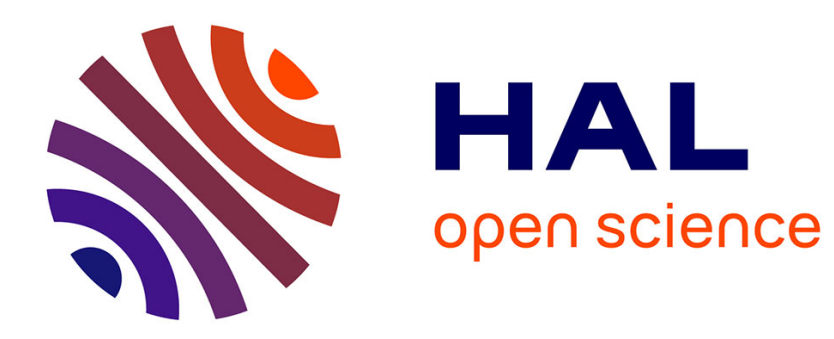

\title{
LE TOURISME PAR LA GÉOGRAPHIE
}

Jean-Christophe Gay

\section{To cite this version:}

Jean-Christophe Gay. LE TOURISME PAR LA GÉOGRAPHIE. Espace Géographique, 2018, 47, pp.97-101. 10.3917/eg.472.0097 . halshs-03550477

\section{HAL Id: halshs-03550477 \\ https://shs.hal.science/halshs-03550477}

Submitted on 13 Feb 2022

HAL is a multi-disciplinary open access archive for the deposit and dissemination of scientific research documents, whether they are published or not. The documents may come from teaching and research institutions in France or abroad, or from public or private research centers.
L'archive ouverte pluridisciplinaire HAL, est destinée au dépôt et à la diffusion de documents scientifiques de niveau recherche, publiés ou non, émanant des établissements d'enseignement et de recherche français ou étrangers, des laboratoires publics ou privés. 


\title{
Le tourisme, approche géographique
}

\author{
Jean-Christophe Gay \\ IAE de Nice \\ Université Côte d'Azur
}

La géographie classique française s'est peu intéressée au tourisme, sans doute parce que dans sa pondération des activités économiques, l'agriculture, l'industrie ou le commerce apparaissaient comme des "postes " plus importants. Albert Demangeon, dans le tome premier sur les îles Britanniques de la Géographie universelle, exprime même de l'incompréhension face au phénomène touristique : " $A$ la fonction urbaine participe un curieux type de villes parasites qui recueillent à certaines saisons l'exode régulier des citadins : aucun pays n'en offre davantage et de mieux constituées que la Grande-Bretagne. Elles apparaissent déjà nombreuses, à la fin du XVIII siècle, sur les côtes ensoleillées de la Manche. [...] II n'est pour ainsi dire pas de citadin qui ne se déplace pour une semaine, au moins une fois par an; les quartiers ouvriers des villes se vident sur les montagnes et sur les plages ; les cités émigrent au bord de la mer, et le sable disparaît sous les foules humaines. " (Demangeon, 1927: 282) Pourtant, ces "villes parasites ", qui représentaient déjà à ce moment-là $5 \%$ de la population anglaise et galloise (Walton, 2000, p. 32), ont mieux résisté au temps qui passe que les villes minières. Il a fallu attendre les années 1950 pour qu'émerge en France une géographie du tourisme qui soit autre chose qu'un simple recensement des équipements, des hébergements ou des fréquentations, ventilés entre mer, montagne, campagne et villes. Des thèses en géographie régionale s'emparent alors du sujet, telles celles de Louis Burnet ${ }^{1}$ sur les côtes françaises (1957), d'Yvette Barbaza sur la Costa Brava (1966), de Micheline Cassou-Mounat sur une partie du littoral aquitain (1977) ou de Daniel Clary sur le littoral normand (1977). Quant à Françoise Cribier (1969) et Jean Ginier (1969), c'est par les mobilités ou les touristes eux-mêmes qu'ils étudièrent le thème. En Allemagne, on peut noter que Walter Christaller (1893-1969) s'est intéressé, dans les années 1950-1960, aux lieux touristiques (Christaller, 1964) parce qu'il a saisi qu'il était difficile de les comprendre par sa théorie des lieux centraux (ibid. :96).

Si le tourisme était un thème subalterne dans la géographie classique française, il l'est largement resté dans tout le champ scientifique. Une telle situation peut s'expliquer par un dédain généralisé pour le tourisme chez les élites économique ou intellectuelle, envisagé généralement sous l'angle de ses conséquences néfastes - qu'il ne s'agit pas de nier ou de minimiser - en matière environnementale ou sociale par exemple. Cette tourismophobie a été précisément disséquée par l'Equipe MIT en 2002 et renvoie à notre difficile relation à la masse, à l'autre, au plaisir ou au repos, dans un monde occidental mettant fortement en avant l'individu et le travail. Sur le marché scientifique, les recherches sur les loisirs restent dévalorisées. Toutefois, la géographie n'est pas la plus mal placée et travailler dans ces domaines n'y est plus stigmatisant. On ne peut pas dire la même chose d'autres disciplines où les recherches et les chercheurs en tourisme sont rares et marginalisés. Ce n'est pas un hasard si nombre de formations universitaires en hôtellerie, restauration, accueil,

\footnotetext{
${ }^{1}$ II est le fondateur, en 1961, et le directeur du Centre d'études supérieures du tourisme à la Faculté des lettres de Paris.
} 
patrimoine, événementiel, etc. sont entre les mains de géographes, qui les orchestrent ${ }^{2}$. II découle de cette implication actuelle des géographes dans la compréhension et l'organisation d'un phénomène économique, social, politique et spatial de très grande ampleur, partie intégrante des sociétés contemporaines, de leurs dynamiques et de leurs recompositions, un fort et rapide renouvellement ainsi qu'une grande diversité des démarches comme, et sans souci d'exhaustivité ou d'ordre, celles portant sur les pratiques, les acteurs, les identités, la géohistoire, le post-colonialisme, le genre, le corps ou les nouvelles technologies.

Le dossier présenté dans ce numéro ne cherche pas à faire le point sur le développement de l'activité touristique aujourd'hui. Par le fait que le tourisme et les loisirs sont actuellement au programme des concours de recrutement de l'enseignement secondaire en histoiregéographie, plusieurs manuels publiés très récemment le font plutôt bien. Ce dossier a été conçu en réaction aux études (cultural studies, gender studies, tourism studies...) qui se multiplient et qui ont tendance à cantonner les disciplines classiques dans un combat d'arrière-garde. Si nous ne rejetons pas ces démarches pluridisciplinaires, souvent très stimulantes, nous croyons également que la recherche à tout à gagner en continuant d'aborder les différentes thématiques par l'angle disciplinaire, parce que, en l'occurrence, les concepts, les théories et les méthodes de la géographie peuvent être enrichis par l'étude $\mathrm{du}$ phénomène touristique. Afin d'illustrer la qualité heuristique $d u$ lien tourisme/géographie, il nous paraissait important de réunir des textes reflétant quelques postulats des approches géographiques actuelles du tourisme. Partant, les articles rassemblés ici, par leur variété, mettent en évidence les stratégies des acteurs sociaux, les processus de diffusion spatiale ou les organisations spatiales micro ou macrogéographiques. Ce n'est peut-être pas un hasard que deux d'entre eux portent sur des territoires asiatiques, cet espace étant aujourd'hui en pleine métamorphose sur le plan touristique, devenant un laboratoire pour analyser des lieux à la fois "mondialisés ", c'est-à-dire qui importent des capitaux, des idées, des innovations sociales, etc., et " mondialisants », c'est-à-dire qui les exportent également (Lévy, 2008).

Dans l'article intitulé "Les logiques de la diffusion du tourisme dans le monde: une approche géohistorique ", nous avons cherché à comprendre, avec Jean-Michel Decroly (Université libre de Bruxelles), comment le tourisme s'est répandu à travers le monde, en définissant le foyer émetteur, en repérant les premiers lieux récepteurs, les cheminements, les vecteurs, les phases, les bifurcations, les ressorts culturels, techniques et économicopolitiques... Quoique la question de la diffusion soit au cœur de la géographie, on peut être surpris et interpelé qu'elle n'ait pas fait l'objet de plus d'attention, car si la propagation des épidémies ou des religions a bien été étudiée, il n'en va pas de même pour nombre d'autres phénomènes, dont le tourisme. Il a fallu faire des choix pour qu'une réflexion sur un sujet de cet ampleur, soit ramenée au format d'un article scientifique, ce qui explique l'importance accordée à la cartographie dans ce travail. La quête des origines du tourisme a ainsi été

\footnotetext{
${ }^{2}$ Notons par exemples en France les cas du premier DESS en tourisme, créé en 1975 à Nice par un géographe, ou de l'Esthua (Etudes supérieures en tourisme et hôtellerie de l'université d'Angers), ex IUP tourisme ouvert en 1993. Devenue l'unité de formation française en tourisme et hôtellerie la plus importante, avec 2900 étudiants, cette dernière a aussi été créée par un géographe et encore aujourd'hui est majoritairement animée par des représentants de cette discipline, puisqu'on y compte treize chercheurs et enseignants-chercheurs géographes contre dix d'autres disciplines.
} 
escamotée, afin de ne pas tomber dans la compétition que se livrent certains historiens pour retrouver les traces les plus lointaines des activités présentes, sans se rendre compte des anachronismes commis, comme le faisait remarquer Pierre Bourdieu lors d'entretiens avec Roger Chartier en 1988 (Bourdieu et Chartier, 2010). Car s'il y a, de l'Antiquité à nos jours, une continuité apparente des mobilités pour son plaisir et sa formation - Montaigne expliquant que la visite des pays étrangers permet de "frotter et limer notre cervelle contre celle d'autrui " (Essais, 1580, livre 1, chapitre XXVI) —-nous considérons, contrairement à certains historiens qui parlent de tourisme à propos de l'Antiquité (André et Baslez, 1993), que c'est dans la seconde moitié du XVIII' siècle et en Angleterre qu'apparaît un nouvel univers social polarisé par une mobilité volontaire et hors quotidienne, généralement divertissante ou/et régénérante. Ce nouveau champ, au sens bourdieusien, que l'on va appeler "tourisme ", se distingue graduellement du Grand Tour en devenant une nouvelle forme d'expérience du monde et en n'étant plus un "modèle d'apprentissage de savoirs politiques mondains et militaires, une pratique de formation qui développe certaines capacités à vivre, à se mouvoir dans le monde, et qui doit être couchée sur le papier » (Bertrand, 2010). Il associe des pratiquants (les touristes) dont les activités vont se diversifier considérablement au fil du temps, des spectateurs séduits (ceux qui aimeraient bien faire du tourisme), des contempteurs (notamment ceux qui se définissent comme des " voyageurs ", cf. Urbain, 1991), des sociabilités, des savoirs et des valeurs, des capitaux, des entreprises, des technologies, etc. II accorde une importance toute particulière aux lieux, dans leurs relations avec nos sensibilités, et porte un nouveau regard sur ceux-ci, à l'origine d'un nouvel usage. C'est la résultante spatiale à l'échelle planétaire de cet ensemble d'éléments, agencés d'une manière caractéristique et nouvelle à partir de la seconde moitié du XVIII siècle, qui sera analysée dans notre texte.

Cette approche macrogéographique de la mondialisation est en contrepoint de l'article d'Emmanuelle Peyvel (université de Bretagne occidentale) et de Marie Gibert (université Paris-Diderot) "La fabrique du quartier routard de Phạm Ngũ Lão (Hồ Chí Minh Ville, Việt Nam). Rapports de pouvoir et inégalités dans la mondialisation touristique " qui l'aborde par le bas, au travers d'une étude de cas minutieuse et inédite portant sur un territoire de cinq hectares, cartographié avec précision à l'échelle cadastrale et associant une spécialiste du tourisme au Vietnam et une géographe urbaniste qui a fait sa thèse sur les ruelles d'Ho Chi Minh Ville. En se plaçant du côté des acteurs locaux impliqués dans l'économie mondialisée du backpacking, les deux auteures nous immergent dans cet univers où les inégalités sociales sont révélées par des micro-organisations spatiales qui les entretiennent. On y découvre l'importance de la logique réticulaire et le mélange de modes de consommation globalisés de loisirs avec des pratiques locales. On est très loin des travaux qui en font des enclaves du tourisme international quand on constate, entre autres, que c'est un lieu de sortie des jeunes Saïgonnais diplômés. Relevant partiellement des études subalternistes par l'intérêt porté aux couches sociales les plus modestes dans une situation post-coloniale, cet article donne une lecture tout en nuances de ce monde aux limites du formel et de l'informel, du légal et de l'illégal, dans lequel les travailleurs du sexe et de la drogue occupent une place non négligeable mais invisible.

C'est aussi une réflexion montrant les relations entre sociétés endogènes et mondialisation du tourisme qui structure le travail de Sylvine Pickel (université d'Angers), de Philippe Violier (université d'Angers) et d'Asep Parantika (Sahid University, Jakarta) intitulé « Le tourisme, 
agent d'interculturation socio-spatiale? Le cas des pratiques touristiques littorales indonésiennes à Bali et Java ». En rupture avec le concept d'acculturation, dominant dans les années 1970-1980, qui ne voyait dans le tourisme qu'un processus d'homogénéisation ou qu'une forme de domination des périphéries par le centre, et donc réfléchissait à son impact sur des sociétés locales jugées incapables de réagir, leur démarche repose sur le concept d'interculturation, qui permet de rendre compte du caractère paradoxal, complexe et indécis du changement culturel dû au tourisme. En introduisant en géographie ce concept, les deux auteurs montrent, dans ce cas indonésien, des formes nuancées de réappropriation des activités balnéaires par les sociétés locales indonésiennes. II faut dire que Bali avait permis à Michel Picard, dans ses travaux pionniers, de démontrer la complexité et la richesse des implications locales du tourisme international ${ }^{4}$. C'est notamment à partir de ce terrain et au travers du tourisme interne, $i$. e. le tourisme pratiqué par les résidents dans leur propre pays, que l'on voit comment le rapport de la société indonésienne au littoral a évolué, construisant son propre " désir de rivage » (Corbin, 1988) tout en révélant l'originalité de ses pratiques de plage, témoignant de la résilience de la culture indonésienne.

La plage est aujourd'hui un des lieux les plus symboliques du tourisme. Chaque année des centaines de millions de personnes de tous âges la fréquentent, s'y amusent, s'y reposent, y lisent, etc. Or elle demeure pour de nombreux géographes un sujet oiseux et il est frappant de remarquer la modestie des travaux qui lui sont consacrés, en regard de sa place dans nos sociétés. C'est pourtant un exceptionnel terrain d'observation et un microcosme des phénomènes que les géographes observent, comme le démontre Peter Haggett (1933-) qui débute son maître ouvrage Geography. A Global Synthesis (2001) par le chapitre liminaire On the Beach. La trentaine de pages que le premier lauréat du prix Vautrin Lud lui consacre sont à la fois une brillante introduction à la pensée géographique et une invitation à étudier ce lieu. Parmi les diverses pistes que donne Peter Haggett, il insiste sur l'étude de la façon dont les plageurs s'installent sur la plage et sur les logiques de leur répartition. Comment ces derniers se placent-ils les uns par rapport aux autres? Comment les cartographier? Comment une plage se remplit-elle et comment analyser la logique de diffusion des plageurs sur celle-ci ? II montre comment on peut mobiliser différents concepts et outils, tel que la time-space geography de Torsten Hägerstrand (1916-2004). L'article « Penser et mesurer les distances de l'interaction sociale dans l'espace de la plage » de Valentin Guyonnard et Luc Vacher (université de La Rochelle) reprend à sa façon la réflexion de Peter Haggett. C'est l'analyse la plus aboutie à notre connaissance sur la question. Mobilisant à la fois de puissants moyens d'observation et des outils sophistiqués de l'analyse spatiale, ils partent de l'interactionnisme d'Erving Goffman (1922-1982) ou de la "proxémie » d'Edward T. Hall (1914-2009) pour cerner l'organisation spatiale des plageurs lors des pics de fréquentation estivale du littoral charentais et pour réfléchir aux distances de la gêne et du confort.

Bibliographie

André J.-M. et Baslez M.-F., 1993, Voyager dans l'Antiquité, Paris, Fayard, 594 p. Barbaza Y., 1966, Le Paysage humain de la Costa Brava, Paris, Armand Colin, 718 p.

\footnotetext{
${ }^{4}$ Nous recommandons la lecture de son témoignage, plus de vingt ans après ses recherches (Picard, 2010)
} 
Bertrand G., 2010, "Grand Tour (tourisme, touriste) " in Christin O. (dir.), Dictionnaire des concepts nomades en sciences humaines, Paris, Métailié, 462 p., p. 171-187.

Bourdieu P. et Chartier R., 2010, Le Sociologue et I'historien, Marseille, Agone, 105 p.

Burnet L., 1957, Villégiature et tourisme sur les côtes de France, Paris, Sorbonne, trois tomes, 502, 792 et $1309 \mathrm{p}$.

Cassou-Mounat M., 1977, La Vie humaine sur le littoral des Landes de Gascogne, Lille, Atelier de reproduction des thèses, deux volumes, 590 et $480 \mathrm{p}$.

Christaller, W., 1964, "Some considerations of tourism location in Europe: The peripheral regions - under-developed countries - recreation areas", Papers in Regional Science, vol 12, $\mathrm{n}^{\circ} 1$, p. 95-105.

Clary D., 1977, La Façade littorale de Paris, Gap, Ophrys, 383 p.

Corbin A., 1988, Le Territoire du vide. L'Occident et le désir du rivage (1750-1840), Paris, Aubier, $411 \mathrm{p}$.

Cribier F., 1969, La Grande Migration d'été des citadins en France, Paris, Editions du CNRS, $403 \mathrm{p}$.

Demangeon A., 1927, Îles Britanniques (tome premier), Paris, Armand Colin, coll. " Géographie universelle », 320 p.,

Ginier J.,1969, Les Touristes étrangers en France pendant l'été, Paris, Genin, 643 p.

Haggett P., 2001, Geography. A Global Synthesis, Londres, Prentice Hall, 833 p.

Lévy J. (dir.), 2008, L'Invention du Monde. Une géographie de la mondialisation, Paris, Presses de Sciences Po, 403 p., p. 393.

MIT, 2002, Tourismes 1. Lieux communs, Paris, Belin, 320 p.

Picard, M., 2010, "L’identité balinaise à l'épreuve du tourisme.", EspacesTemps.net, Travaux, 12.4.2010

https://www.espacestemps.net/articles/identite-balinaise-epreuve-tourisme/

Urbain J.-D., 1991, L'ldiot du voyage. Histoires de touristes, Paris, Plon, 275 p.

Walton J. K., 2000, The British seaside. Holidays and resorts in the twentieth century, Manchester, Manchester University Press, $216 \mathrm{p}$. 